\title{
EPSL
}

ELSEVIER

Earth and Planetary Science Letters 183 (2000) 389-401

www.elsevier.com/locate/epsl

\section{Paleointensity record in zero-age submarine basalt glasses: testing a new dating technique for recent MORBs}

\author{
J. Carlut ${ }^{\mathrm{a}, *}$, D.V. Kent ${ }^{\mathrm{a}, \mathrm{b}}$ \\ a Lamont-Doherty Earth Observatory, Paleomagnetics Laboratory, Route 9W, Palisades, NY 10964, USA \\ b Department of Geological Sciences, Rutgers University, Piscataway, NJ 08854, USA
}

Received 4 August 2000; received in revised form 28 September 2000; accepted 28 September 2000

\begin{abstract}
Thellier-Thellier paleointensity experiments were conducted on a collection of glasses from three very recent submarine axial flows. Two were erupted along the Juan de Fuca ridge at around $46^{\circ} \mathrm{N}$ and one along the East Pacific Rise South at around $18^{\circ} \mathrm{S}$. The within-sample dispersion of paleointensity results from the 'Animal Farm' flow (EPR south) is very low and leads to a well-defined mean value of $35.6 \pm 1 \mu \mathrm{T}$ ( $95 \%$ error on the mean) based on 11 glass chips from four independent samples. Today's geomagnetic field intensity in the area is $31.2 \mu \mathrm{T}$. Comparing Animal Farm results with published field model reference curves developed for the past $400 \mathrm{yr}$ suggests an eruptive date estimated between 1880 A.D. and 1950 A.D. (taking into account different sources of errors). This is consistent with qualitative evidence for the age of this flow and constitutes the first precise demonstration of using paleointensity as a dating tool for very recent mid-ocean ridge basalts (MORBs). However in the Juan de Fuca area results show a more erratic pattern with samples varying by up to $30 \%$ higher and lower from the expected value of about $55 \mu \mathrm{T}$. The dispersion is attributed to the large local crustal magnetic anomalies in this area that can lead to inconsistent intensity values over the same unit. Local magnetic anomalies should thus always be checked when doing paleointensity on MORB samples which should also be distributed as widely as possible in a flow unit. When no significant magnetic anomalies are detected the paleointensity dating tool is anticipated to be especially efficient to investigate the volcanic cyclicity along the EPR axis during the last several hundred years. (C) 2000 Elsevier Science B.V. All rights reserved.
\end{abstract}

Keywords: magnetic intensity; paleomagnetism; geochronology; mid-ocean ridge basalts; East Pacific Rise

\section{Introduction}

Dating of submarine lavas is notoriously difficult (e.g. [1]). Several isotopic methods have tentatively been applied. A promising chronometer based on $\mathrm{Po}-\mathrm{Pb}$ radioactive disequilibrium can

\footnotetext{
* Corresponding author. Fax: +1-914-365-8158; E-mail: jcarlut@1deo.columbia.edu
}

date samples of mid-ocean ridge basalt a few years old [2] and U-series dating techniques have been used to date samples on time scales of the last few 1000 to several $10000 \mathrm{yr}[3,4]$. However these $\mathrm{Po}-\mathrm{Pb}$ and $\mathrm{U}$-series dating techniques are rather delicate and do not cover the critical last several tens to hundreds of years corresponding to the expected fast to intermediate recurrence interval of magmatic events. Consequently, the ages of exposed lavas in the axial region have been estimated mainly on the basis of in situ observations 
(sediment cover, degree of alteration, presence and maturity of hydrothermal sites and their biological communities; see as an example Ken Macdonald's Relative Age Scale for lavas on the EPR $\left.\operatorname{axis}^{1}\right)$. These techniques are very qualitative and dependent on our very partial understanding of the volcanological, tectonic, hydrothermal and biological processes at the ridge axis.

The geomagnetic dipole field has been decreasing in magnitude at the rate of about $5 \%$ per century over at least the past several hundred years [5]. Over longer time scales, the geomagnetic field varied by nearly an order of magnitude, from about 50\% higher than today's field about $2000 \mathrm{yr}$ ago to less than $25 \%$ about 30000 yr ago [6]. The great potential of paleointensity analysis on submarine basalt glass [7] as a recorder of past geomagnetic variations and a dating tool motivates us to evaluate the accuracy and resolution of paleointensity estimate on very recent submarine basalts.

Although mid-ocean ridge basalts (MORBs) are likely to experience low temperature oxidation, high resolution electron microscope analyses conducted on zero-age as well as more than $30 \mathrm{Ma}$ old oceanic basalts $[8,9]$ reveal that interstitial glasses do not seem to be sensitive to alteration on such time scales. A recent study by this group shows that the outer-rim glasses appear identical to the interstitial glasses and have not experienced alteration, at least over recent times (see [10]). Furthermore, several studies have shown that the glasses have magnetic properties consistent with a thermoremanent magnetization carried by very fine-grained (titano-) magnetites in the single domain state (see $[8,9,11,12]$ ). MORB glasses should therefore be ideal material for paleointensity measurements (e.g. [13]). There has been very recently an increasing number of studies reporting experiments using submarine glasses for paleointensity purposes $[7,14,15]$. Three recently published studies show that results obtained from newly erupted flows (which thus cooled in a known field) can give accurate values $[7,15,16]$.

\footnotetext{
${ }^{1}$ http://imina.soest.hawaii.edu/at1331/KenMacdonaldAge Scale.html
}

We seek further supportive evidence for the use of glassy or near glassy MORB samples for paleointensity measurements and ultimately dating.

\section{Sample and experimental procedure}

In June 1993 the eruption of a new flow along the Juan de Fuca ridge was recorded by the SOSUS network (US Navy's SOund SUrveillance System) installed close to the ridge axis in the

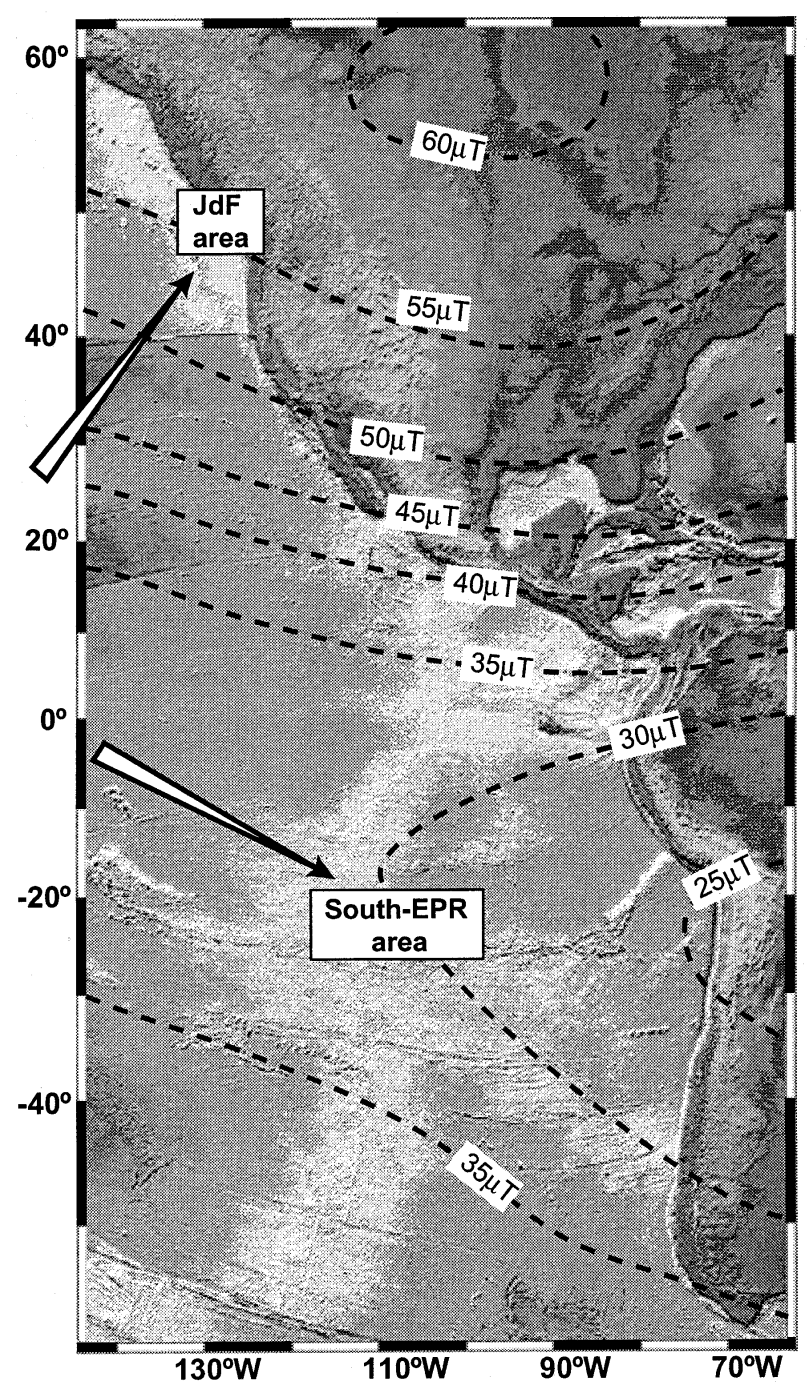

Fig. 1. General map of the EPR with the Juan de Fuca coaxial segment at $45^{\circ} 50^{\prime}-46^{\circ} 30^{\prime} \mathrm{N}$ and Animal Farm at $18^{\circ} \mathrm{S}$ area sites (after [36]). Isodynamic contours for 2000 A.D. showing the variations of magnetic field intensity over the Earth's surface are also represented. 
North East Pacific (see e.g. [17] for a description). The event was precisely located (near $46^{\circ} 31.5^{\prime} \mathrm{N}$; $129^{\circ} 35^{\prime} \mathrm{W}$ ) allowing the sampling by submersible of this new flow soon after the eruption. Three independent samples from the 1993 flow, located at a few hundred meters away from each other were obtained for this study: Turtle 787-20-94 and ATV 94-50-94R2, hereinafter named R1-93 and R2-93, respectively (pillow samples provided by H.P. Johnson; R1-93 has previously been studied by Kent and Gee [16]), and 2792-1R2 named R3-93 (glassy fragments provided by M. Perfit and J. Chadwick). In 1998, a new eruption was recorded south (lat $\sim 45^{\circ} 56^{\prime} \mathrm{N}$; lon $\sim 129^{\circ} 59^{\prime} \mathrm{W}$ ) of the 1993 event (e.g. [18]). Two samples some tens of meters apart R462-8 and R462-15 (glassy fragments provided by M. Perfit and J. Chadwick) referred to in the following as R1-98 and R2-98, respectively, were obtained for this study and added to this 'zeroage' Juan de Fuca dataset (see Fig. 1).

Four additional samples from a lava flow in a different area, the Southern East Pacific Rise $\left(18^{\circ} \mathrm{S}\right.$ area; see Fig. 1), were also analyzed. They were collected at some hundreds to thousand meters apart by the Alvin submersible. The recent age of the flow was attested to by the shimmering water emerging from the surface observed during a dive made in December 1993 (see [19,20,21]). The flow was at that time densely colonized by vent animals and subsequently named 'Animal Farm' (abbreviated AnF in the following). These lobate and pillow samples were provided to us by Milene Cormier.

Sub-samples of small glassy chips from the outer part of each fragment were used for Thellier experiments. Typical glassy chips weigh only a few tens of milligram. We selected sub-samples whose total magnetic moment is greater than $5 \times 10^{-10} \mathrm{Am}^{2}$ which we regard as a threshold for reliable results (i.e. at least two orders of magnitude greater than the noise level of our cryogenic magnetometer). The total moment distribution of all samples is shown in Fig. 2; most have a total moment between $10^{-9}$ and $10^{-8} \mathrm{Am}^{2}$. Due to the small size and weak magnetization of the chips, we were especially careful regarding the background noise level, including contamination

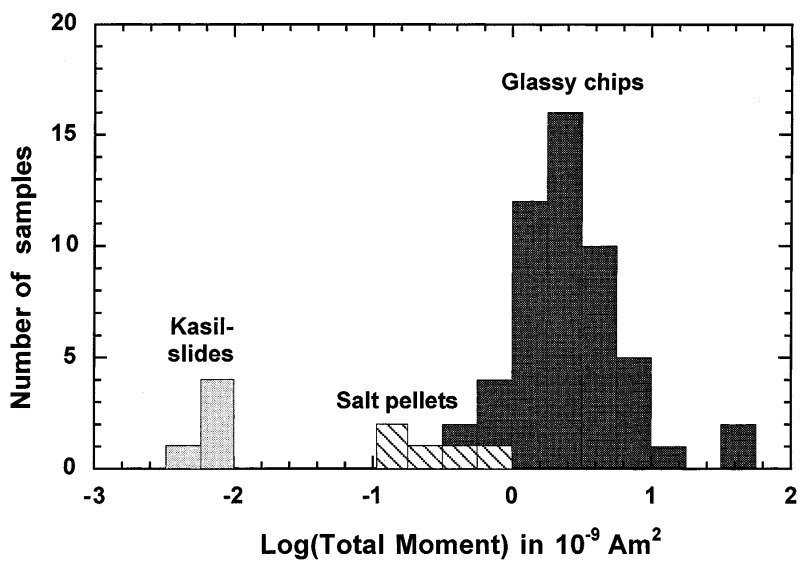

Fig. 2. Histogram of total magnetic moments of 52 MORB glassy chips, five representative blank salt pellets and five representative blank microscope slides.

from the holder and experimental drift while measuring. The irregular shape of the small chips requires they be somehow fixed to a mounting medium to allow the necessary precise control of their orientation during the experiment. Of particular concern was the magnetization of this mount. An initial experiment was conducted with R2-93 chips using the salt encapsulation method described by [14] (see also [15,22]). All subsequent experiments were made using chips mounted with a drop of Kasil on microscope glass slides (described below).

The modified Thellier double heating method [23] was used. Samples were heated and cooled in air. Three experiments were made, one with a laboratory field of $33 \mu \mathrm{T}$ and two with a field of $40 \mu \mathrm{T}$. Back-checks were obtained after every other temperature step in order to document any magneto-mineralogical changes that might be produced during the laboratory heating. Remanence measurements were made using a $2 \mathrm{G}$ DC-Squid magnetometer. The results are presented in the classical Arai plot [24] where magnetization remaining is plotted as a function of the acquired magnetization. A result is considered acceptable if the ratio between lost and acquired magnetization is nearly constant over a wide range of NRM demagnetization where a single direction component is determined. Back-checks must lie within $5 \%$ of the original p-TRM indicating the absence of alteration during the course of the experiment. 
The fact that the geomagnetic field intensity was precisely known for the time of eruption of the flows at the $\mathrm{JdF}$ segment (IGRF $=53.7 \mu \mathrm{T}$ for the 1993 coaxial flow, $52.9 \mu \mathrm{T}$ for the axial 1998 flow; see Fig. 1) allowed us to evaluate the reproducibility and ultimately the accuracy of the paleointensity results from the submarine glasses. Similarly, in situ observations, indicating a fairly recent eruption for the AnF samples, leads us to expect results close to today's field at the $18^{\circ} \mathrm{S}$ locality ( $\sim 32 \mu \mathrm{T}$; see Fig. 1$)$. To estimate the secular variation in Earth's magnetic intensity, the IGRF and DGRF for recent time (i.e. after 1900 A.D.), and the spherical harmonic model of Bloxham and Jackson [5] until degree and order 8 between 1700 and 1900 A.D., were used to calculate expected values for the exact sampling locations.

\section{Magnetic mineralogy}

The NRM intensities of the chips are low and range between $5 \times 10^{-6} \quad \mathrm{Am}^{2} / \mathrm{kg}$ and $1 \times 10^{-4}$ $\mathrm{Am}^{2} / \mathrm{kg}$. The maximum unblocking temperature of the chips is typically reached between $430^{\circ} \mathrm{C}$ and $575^{\circ} \mathrm{C}$. The magnetic hysteresis parameters

a)

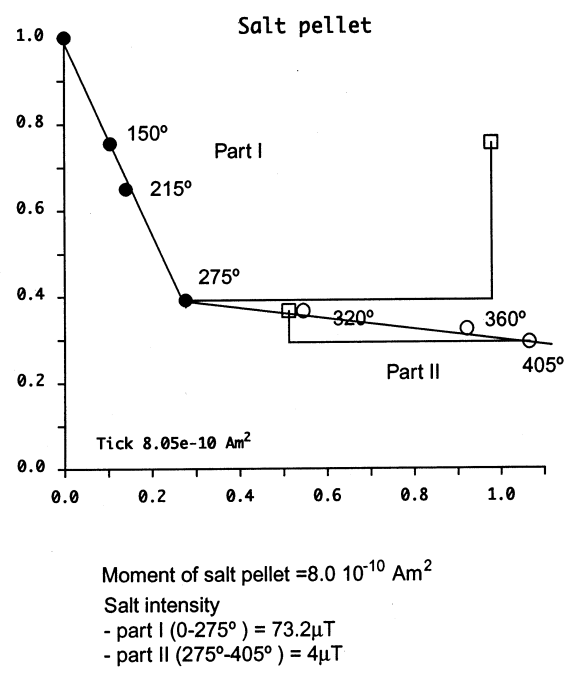

$M_{\mathrm{r}}, M_{\mathrm{rs}}, B_{\mathrm{c}}$ and $B_{\mathrm{cr}}$ have been measured for a representative set of glass chips from the same sample used for paleointensity. Results show high coercivities $\left(B_{\mathrm{cr}}\right.$ often above $\left.100 \mathrm{mT}\right)$ and low to very high $M_{\mathrm{rs}} / M_{\mathrm{r}}$ ratio (from close to 0.1 up to 0.7 ). These observations are typical for such very fine-grained rocks as already discussed by several authors (see for example $[9,12,16,25]$ ). Elevated Curie temperatures near the pillow margin were once taken as evidence of low temperature alteration (e.g. [16]) but detailed mineralogical studies that included transmission electron microscopy by Zhou et al. [10] have shown that the MORB rim glasses reflect a magnetic population of unaltered very fine-grain (partly in the super-paramagnetic range) titanomagnetite grains where the broad range of unblocking temperature is related to the broad range of ulvospinel compositions ( $x$ is between 0 and 0.45 ) encompassed by the carrier of magnetization.

\section{Mounting technique}

Initial experiments were made using a salt encapsulation pellets technique (e.g. [14,15,22]). Twelve chips from R2-93 were measured and

b)

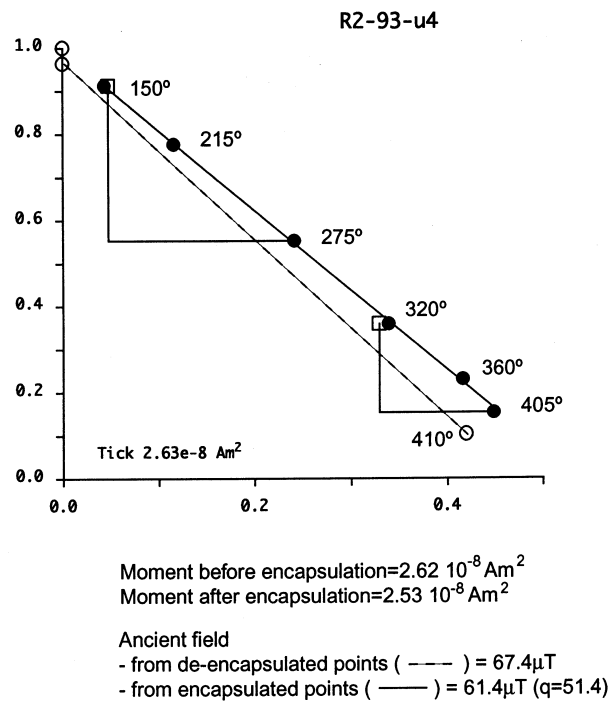

Fig. 3. NRM versus TRM (Arai) diagrams of Thellier-Thellier results obtained using the salt encapsulation technique. Circles show NRM-TRM values. Also shown are the p-TRM checks (squares). In (a) a salt pellet is measured showing its ability to acquire magnetization. In (b) a comparison of paleointensity values obtained using steps made with the encapsulated chips (plain lines) and disencapsulated chips (first and last steps, dashed lines). 
then encapsulated with common salt $(\mathrm{NaCl})$ in a hydraulic press at 8000 psi into cylindrical pellets having a diameter of $\sim 2.5 \mathrm{~cm}$ and a length of $\sim 1 \mathrm{~cm}$. Several 'blank' salt pellets were prepared and measured during the Thellier experiments at the same time.

Approximately $30 \%$ of encapsulated chips show signs of poor behavior during the course of the Thellier experiment (no linear relationship between lost and gained magnetization and/or negative p-TRM checks at relatively low temperatures). Successful results showed a large dispersion (standard deviation of $8 \mu \mathrm{T}$ ) around a mean of $54.5 \mu \mathrm{T}$. Although the salt magnetic moment is quite low, it is sometimes within only one order of magnitude of the mean initial magnetic moment of the MORB glass chips (see Fig. 2). Furthermore, the blank salt samples show that a magnetization can be acquired by the salt during the p-TRM step (Fig. 3a). It is therefore likely that contamination of the chip magnetic signal by salt encapsulation occurred. This was confirmed by calculating the slope obtained using the first measurement made before encapsulation and a last NRM/TRM step made after disencapsulating the chips (by dissolving the salt pellets in water). The difference between the respective slopes is clearly apparent on Fig. 3b. It is interesting to note that even when the first double heating step shows no sign of significant contamination, a bias can clearly develop in subsequent steps. This effect preferentially biases the result toward low paleointensity values because the salt is more likely to gain magnetization than to lose any weak initial signal.

Considering the above results, we developed an alternative mounting technique to reduce potential magnetic contamination of the weakest glass chips. Chips are mounted with Kasil (potassium silicate) on cleaned microscope slides which allows the chips to be easily handled and oriented (Fig. 4). Measurements confirm that the initial magnetization of the slides is negligible (total magnetic moment is less than $10^{-11} \mathrm{Am}^{2}$; magnetization is less than $5 \times 10^{-9} \mathrm{Am}^{2} / \mathrm{kg}$ ), due to a combination of smaller total mass and higher purity than the salt pellets (whose total moment is on the order of $5 \times 10^{-10} \mathrm{Am}^{2}$ and magnetization reach

\section{Kasil - slide method}

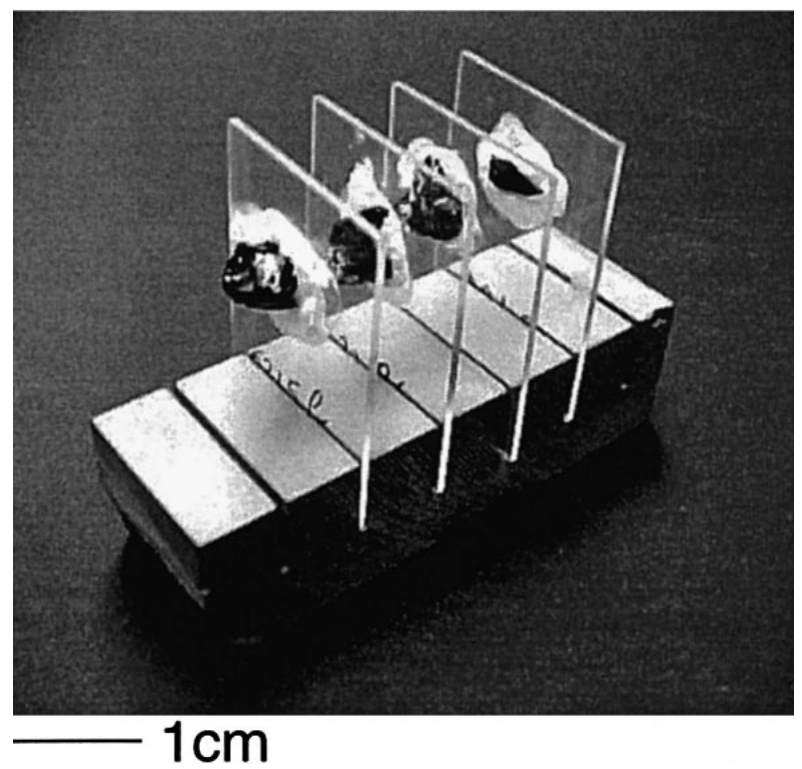

Fig. 4. Photograph showing the Kasil-slide technique.

$5 \times 10^{-8} \mathrm{Am}^{2} / \mathrm{kg}$; see Fig. 2). Moreover no pTRM was acquired during in-field heating and cooling (an example is given in Fig. 5f). All the following results are obtained using this Kasilslide method, which also involves a slotted holder for the oven and a slotted ring for measurements (see Fig. 4).

\section{Results and comparison with the IGRF}

A total of 52 MORB glassy chips were measured. Fifteen sample chips come from Animal Farm and 37 come from the Juan de Fuca area (30 from the 1993 flow and seven from the 1998 flow).

Most chips are characterized by a single welldefined magnetization component. In a few cases the glassy chips from closest to the chilled margin show an initial low unblocking temperature component. Considering that the outermost samples are cooled to below the Curie temperature in less than a minute (from Carslaw and Jaeger, 
(a)

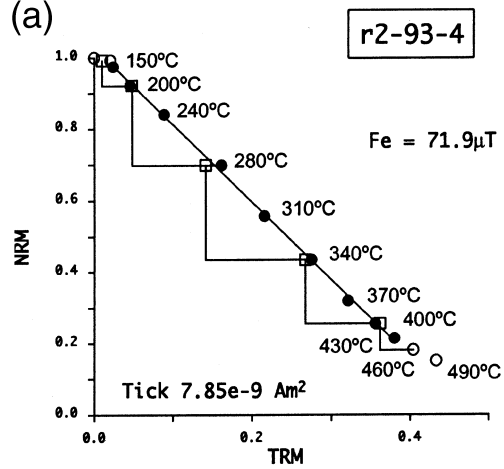

(b)

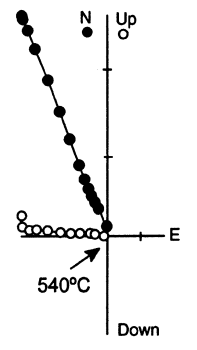

(c)

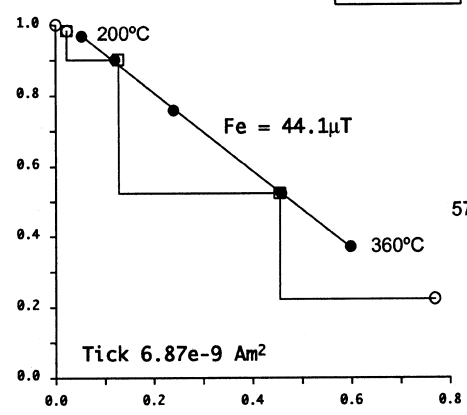

(e)
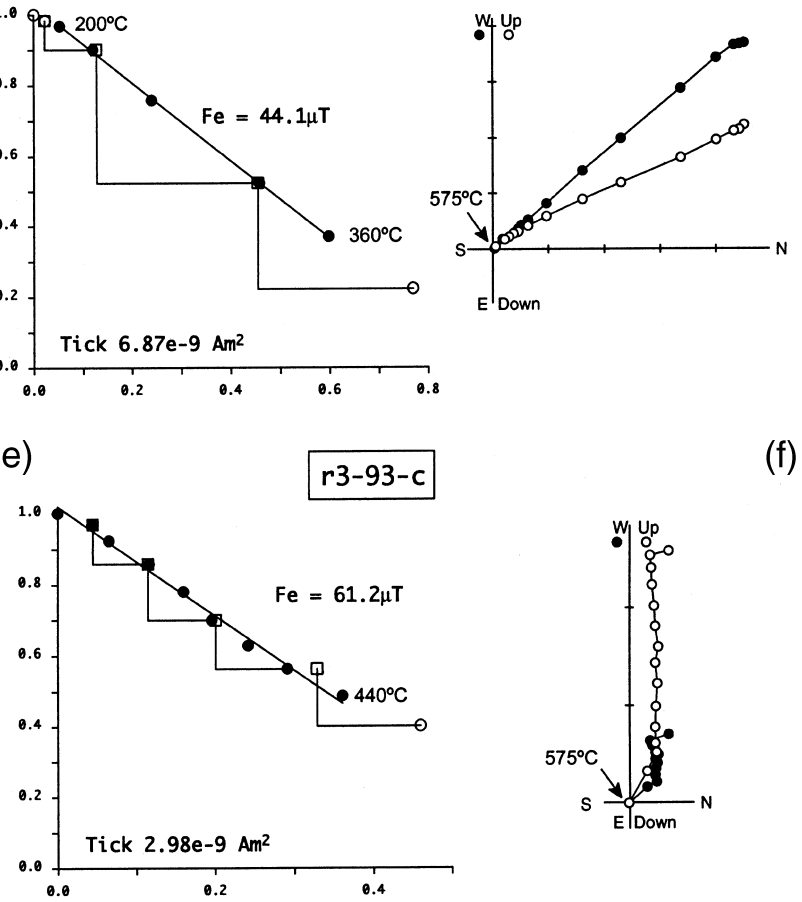

(f)

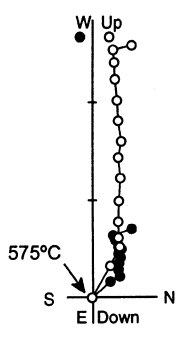

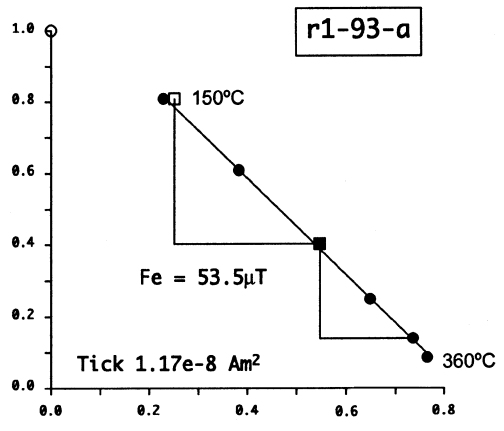

(d)
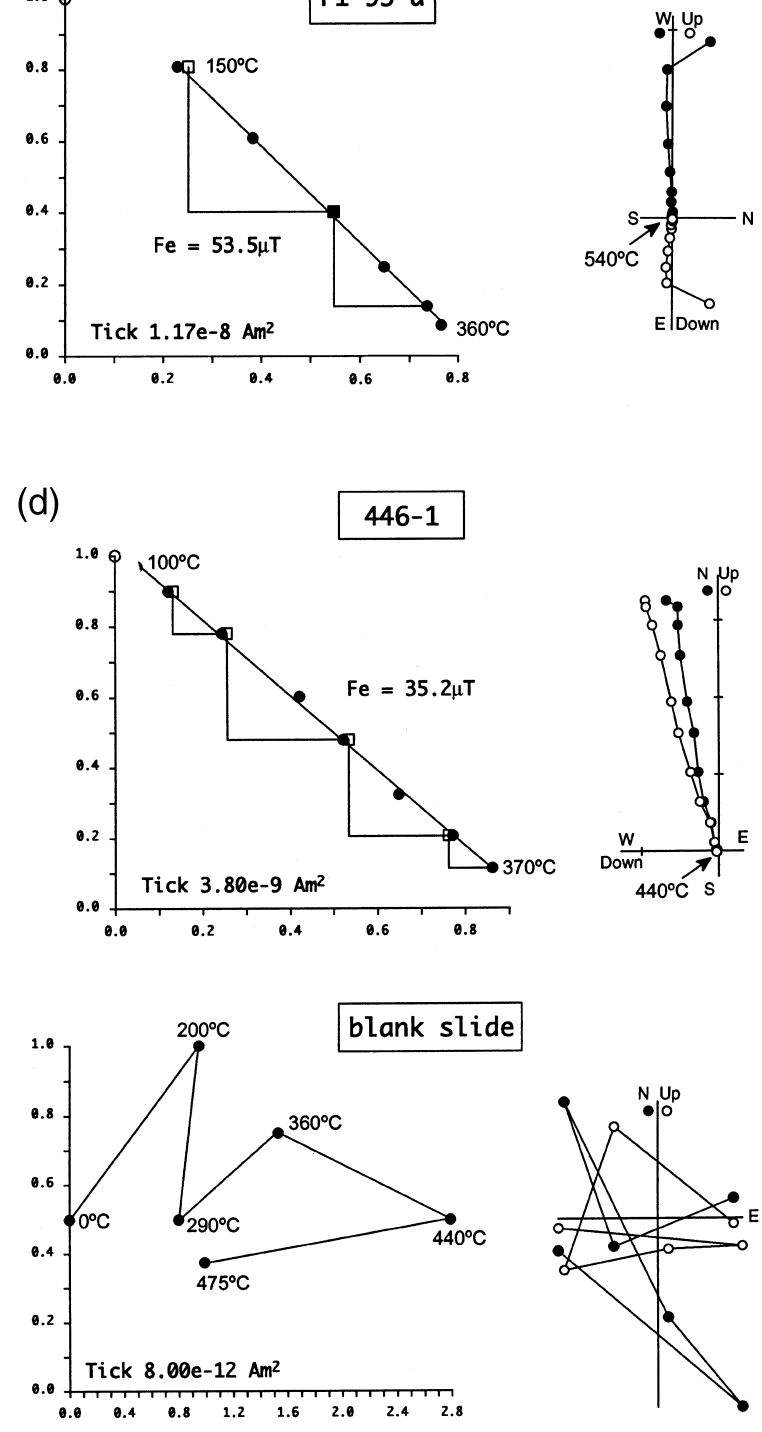

Fig. 5. NRM versus TRM (Arai) diagrams for typical samples from all flows (a-e) and a blank microscope slide (f). Included for each sample are Zijderveld [37] diagrams, solid symbols corresponding to projections onto the horizontal plane, while open symbols are projections onto the vertical plane. All results shown use the microscope slide technique.

[26]), this component probably reflects acquisition during inflation (and rotation?) of the outer surface of the flow as the erupting magma is still being emplaced. During the paleointensity experiments the majority of chips showed good behavior: nearly constant ratio between lost and acquired magnetization and positive back-checks. Representative results of the experiments are shown in Fig. 5 and a summary of all results is presented in Table 1. These experiments are characterized by a high overall success rate (on the order of $80 \%$ ) and high $q$ factors of individual paleointensity determinations $(q$ is here often above 30 , see $[27,28]$, for a definition) which reflect the high internal consistency of the analyses. 


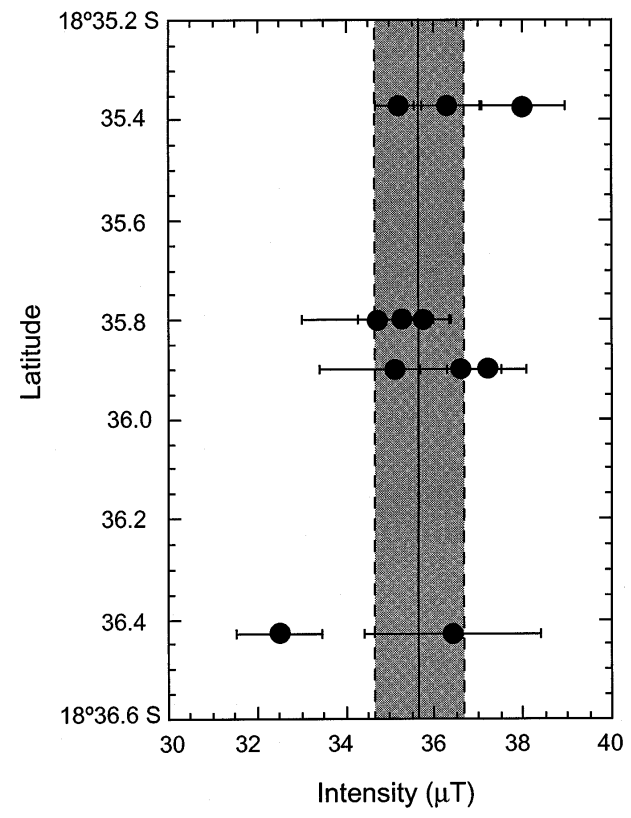

Fig. 6. Individual paleointensity results obtained from the Animal Farm flow at $18^{\circ} \mathrm{S}$ versus sample site latitude. Error bars are associated standard errors, line and shaded area are overall mean value and $95 \%$ standard error on the mean, respectively.

\subsection{Results from $18^{\circ} \mathrm{S}$ area}

Eleven out of 15 chips analyzed from the Animal Farm flow show acceptable behavior (mean $q$ around 30 , back-checks closely matching previous TRM). The four lobate pieces give results between $36.5 \pm 1.4 \mu \mathrm{T}$ and $34.4 \pm 2.7 \mu \mathrm{T}$ (Fig. 6 and Table 1) with an overall mean of $35.6 \pm 1.0 \mu \mathrm{T}$ (calculated giving each lobate pieces the same weigh). The dispersion around the mean is very low leading to a high precision determination of the overall paleointensity (with a resolution better than $5 \%)$.

The AnF flow is described as recent, some 10 to $100 \mathrm{yr}$ old, although partially covered by a very young flow [21]. The reference curve for the last $300 \mathrm{yr}$ (after [5]) projected at $18^{\circ} \mathrm{S}$ is represented on Fig. $7 \mathrm{a}$ and shows a decrease of $10 \mu \mathrm{T}$ or about $25 \%$ over this time interval. According to this reference curve, the AnF flow paleointensity value should correspond to a fairly recent time of eruption (1910 \pm 20 A.D., using formal 95\% confidence limit). This result is thus in agreement with the in situ observations.

\subsection{Results from Juan de Fuca}

The extremely well-dated 1993 and 1998 flows from the $\mathrm{JdF}$ area represent a unique opportunity to test the reproducibility of the encouraging results found from the $18^{\circ} \mathrm{S}$ area in a different context (higher geomagnetic field intensity, different chemistry of the lavas).

For the 1993 flow, the successful paleointensity experiments from R1-93 (seven of ten chips) give
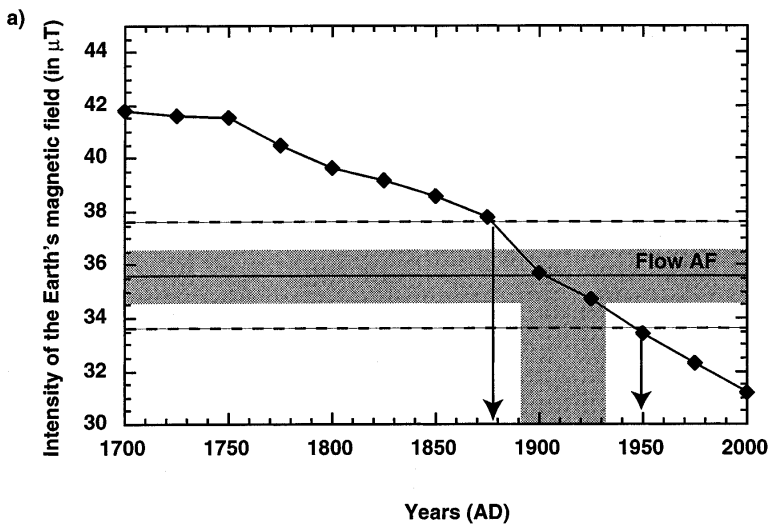

b)

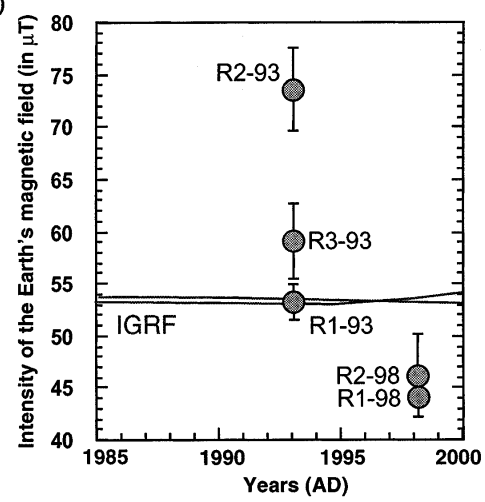

Fig. 7. Comparison between the paleointensity results (error bars are 95\% standard error) obtained and paleofield reference curves. (a) Results from the $18^{\circ} \mathrm{S}$ area. The reference curve has been projected at the Animal Farm site $\left(18^{\circ} 36^{\prime} \mathrm{S}\right.$; $113^{\circ} 27^{\prime} \mathrm{W}$ ) and made using DGRF back to 1900 A.D. then the spherical harmonic model of Bloxham and Jackson [5] from 1700 A.D. to 1900 A.D. The AnF flow paleointensity result suggests the flow was emplaced in the early part of the 20st century taking into account the additional $1 \mu \mathrm{T}$ error due to local field (dashed lines). (b) Results from the JdF area. The last 15 years of intensity variations at the 1998 and 1993 flow sites (solid lines) are derived using the IGRF. A strong dispersion of results is observed around the expected value $(\sim 54 \mu \mathrm{T})$. 


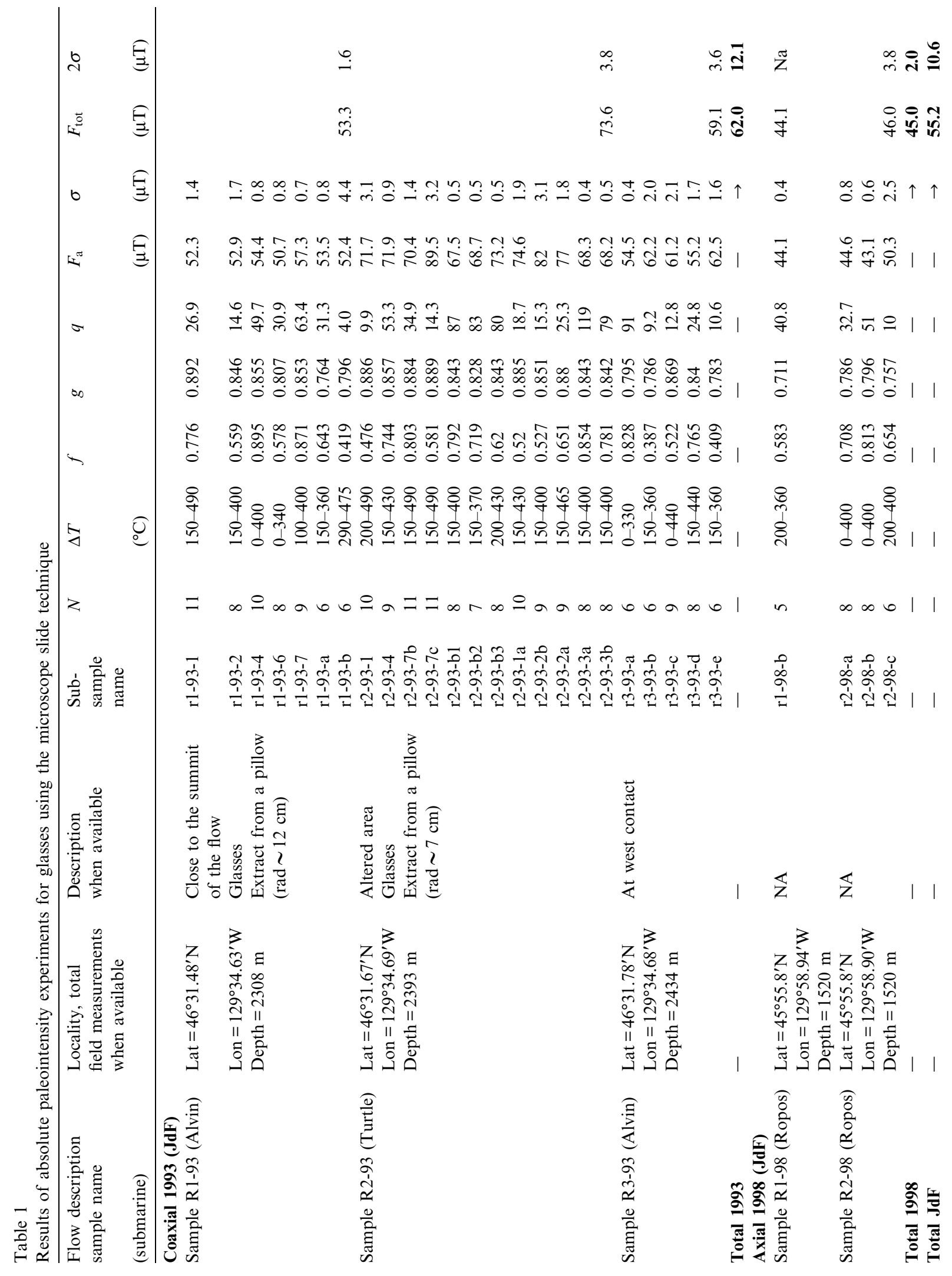


J. Carlut, D.V. Kent/Earth and Planetary Science Letters 183 (2000) 389-401

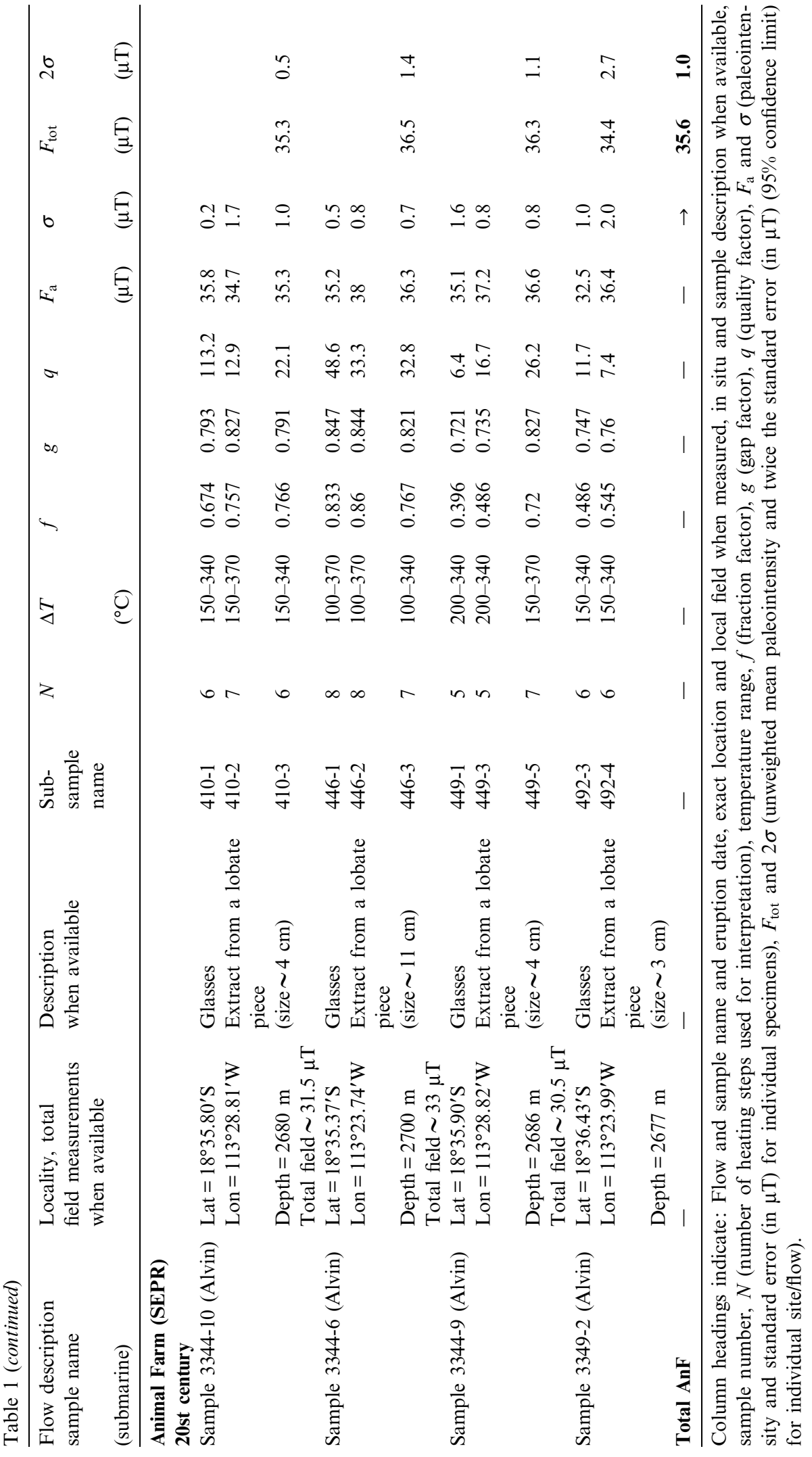


a mean value of $53.3 \pm 1.6 \mu \mathrm{T}$. The successful paleointensity experiments from R3-93 (five of five chips) give $59.1 \pm 3.6 \mu \mathrm{T}$, somewhat higher although not significantly so compared to R193. To our surprise, R2-93, which was reported to be from the same 1993 flow, shows a mean intensity of $73.6 \pm 3.8 \mu \mathrm{T}$ (based on 12 chips), more than $20 \%$ higher than values from the other two samples.

Seven chips from two distinct pieces from the 1998 eruption were measured. Three chips were discarded due to strong multi-component magnetization visible in the vector end-point diagram and/or negative p-TRM checks. The remaining four chips give well-grouped paleointensities around a mean of $45.0 \mu \mathrm{T}$ (with a $95 \%$ confidence limit of $2.0 \mu \mathrm{T}$ ), significantly lower than the samples from the 1993 flow.

The mean value of $53.3 \pm 1.6 \mu \mathrm{T}$ obtained for R1-93 is in perfect agreement with what is inferred from IGRF at this location during the eruption in 1993 (53.7 $\mu \mathrm{T}$; see Fig. 7b). Sample R3-93 gives a value a little bit too high $(59.1 \pm 3.6$ $\mu \mathrm{T})$. However the high and well-defined value of $73.6 \mu \mathrm{T}$ obtained for R2-93 is unexpected, as is the low value of $45.0 \mu \mathrm{T}$ obtained for the 1998 axial flow (IGRF at this place gives $52.9 \mu \mathrm{T}$ for 1998; see Fig. 7b).

\section{Discussion}

The AnF data from $18^{\circ} \mathrm{S}$ are highly consistent both internally and with respect to independent evidences of a young age for this flow. This result demonstrates that an analytical resolution on the order of $3-4 \%$ (95\% standard error of the mean) is achievable when dealing with paleointensity determination of MORB glasses. It can probably be considered as a practical threshold of uncertainty as fundamental problems arise from the choices of $\mathrm{NRM} / \mathrm{p}$-TRM points to be included in the slope calculation of the Arai diagram. The paleointensity data from the $18^{\circ} \mathrm{S}$ samples also suggest that the magnetization carried by outer-rim glasses is indeed a TRM, since a CRM would not be expected to produce coherent data from sample to sample. The AnF result demonstrates the practic- ability of a dating method based on paleointensity analysis for recent submarine flows. For the past $500 \mathrm{yr}$, the resolution of the paleointensity method should be similar to the predicted time frame for eruptive cycles at fast spreading ridges of about $50 \mathrm{yr}$. This is sufficient to date the neovolcanic zone on the EPR and needed to constrain the timing and thus the emplacement mechanism of lavas along the axis. For samples from the last several thousands years, this method can also be used as a tool to differentiate products from successive volcanic eruptions.

In contrast, a more erratic pattern was encountered in the JdF area even though there is high certainty in the ages of the sampled flows. The within-sample dispersion is comparable to $\mathrm{AnF}$ for R1-93 and to a lesser extent R3-93, but is significantly noisier for sample R2-93. The origin of dispersion between chips from the same sample is not well understood since no particular problems were detected during the course of these experiments and the positive p-TRM checks indicate the absence of alteration. Still it can be noted that the chips from R2-93 giving the (anomalously) highest values also give the highest standard deviation for the Arai slope and thus are of lower quality. The other samples presented here do not suffer from the same noisy behavior but the between-sample scatter is nevertheless high, with values ranging from 44.1 $\mu \mathrm{T}$ (1998 flow) to $73.6 \mu \mathrm{T}$ (1993 flow). The mean for the 1993 flow (obtained on three samples) is $62 \pm 12.1 \mu \mathrm{T}$. If the two samples from the 1998 flow are also included the overall mean is $55.2 \pm 10.6 \mu \mathrm{T}$. Although the overall mean value for the 1993 and 1998 flows happens to be close to the expected geomagnetic field intensities $(52.9-53.7 \mu \mathrm{T})$, the large scatter in the paleointensity results does not lend great confidence for precise dating in this region.

One explanation for the scatter is that the JdF samples may not be well located and therefore could actually not be part of the same flow. Precise contacts between flows are not easy to map: for instance, determination of lava flow edge using SeaBeam bathymetry is only at some 5 to $15 \mathrm{~m}$ resolution [29]. However, this would hardly explain low paleointensity values from the axial region since the geomagnetic field has 
been higher than the present for the past several kiloyears [6] implying the flows at the axis would have to be older than this which is not a plausible option.

Another explanation is that local crustal magnetic fields could add a highly variable contribution to the geomagnetic field during the initial cooling of lava flows, especially in the JdF area. Several near bottom magnetic profiles were collected close to the area where the samples were taken along the EPR at $18^{\circ} \mathrm{S}$ and the 1993 flow in the $\mathrm{JdF}$ area. Anomalies on the order of only 1 or $2 \mu \mathrm{T}$ (10-100 $\mathrm{m}$ above bottom) were measured in the $18^{\circ} \mathrm{S}$ area [30,31]. A different pattern is observed along tracks collected over the 1993 coaxial flow (some $10 \mathrm{~m}$ above bottom) showing significant magnetic anomalies up to $10 \mu \mathrm{T}$ $[32,33]$. Inconsistent intensity values would result if such high magnetic anomalies were present during emplacement of the 1993 (and 1998) flows.

The higher magnetization contrast responsible for the intense anomalies at the JdF site compared to the $18^{\circ} \mathrm{S}$ EPR area is mainly explainable by (1) higher total iron contents in the $\mathrm{JdF}$ area (e.g. $\sim 13.0 \mathrm{wt}^{\%} \% \mathrm{FeO}^{*}$ in 1993 basalt, M. Perfit, personal communication) compared to the $18^{\circ} \mathrm{S}$ area (average value of $\sim 11 \mathrm{wt} \%$ in the $18^{\circ} \mathrm{S}$ EPR area [34]), and (2) the higher field intensity at the JdF latitude (today's field is $\sim 54 \mu \mathrm{T}$ for the 1993 flow site compared to $\sim 32 \mu \mathrm{T}$ for AnF site). These observations support the hypothesis that short wavelength crustal magnetic anomalies are an important cause of inconsistent intensity values in units from the JdF area. This explanation implies that the magnitude of local anomalies should be documented when dealing with paleointensities of MORB at the ridge axis and included in error bars. For the AnF the high reproducibility of paleointensity values between samples from different parts of the flow (Table 1; Fig. 6) confirms the low crustal magnetic anomaly in the area (see also direct measurements made by the Alvin, Table 1). Nevertheless a $1 \mu \mathrm{T}$ error bias might be included in the error budget, leading to a more conservative estimate for the AnF eruptive date between 1880 and 1950 A.D. (see Fig. 7a).

\section{Conclusions}

Paleointensity analyses using the Thellier-Thellier method were conducted on volcanic glasses from three very recent submarine flows. A new technique, in which samples are mounted with Kasil glue on microscope slides, allows us to achieve a high level of analytical precision with small $(\sim 100 \mathrm{mg})$, irregular, and very weakly magnetized glass chips.

Analyses of the Animal Farm flow on the EPR at $18^{\circ} \mathrm{S}$ shows that paleointensity values can be obtained from within a flow with a high reproducibility. These results added to previous studies $[7,14,15]$ show that volcanic glasses are potentially a good recorder of the Earth's magnetic field intensity. The recent emergence of global reference curves of intensity variations for the last several hundred years [35] provides a basis to estimate an eruptive date for young flows. Tests in the Juan de Fuca area $\left(46^{\circ} \mathrm{N}\right)$ show unexpected discrepancies within samples from the same flows. Some results are up to $30 \%$ higher than expected, others up to $20 \%$ lower. This is in contrast with the wellclustered results found in the $18^{\circ} \mathrm{S}$ locality. The most plausible interpretation is that strong local magnetic anomalies add a contribution to the main field. The dispersion in paleointensity from 1993 and 1998 flows implies that large magnetization contrasts existed prior to the emplacement of these flows. Therefore, magnetic anomalies may not be closely related to any individual flow (see e.g. [33]).

These results emphasize that paleointensity studies on MORBs should be combined with near bottom magnetic surveys and that the mean paleointensity value for a flow unit should be determined using several samples taken at different locations within this flow. The magnitude of local crustal anomalies can then be included in the error budget. This is most obvious in the Juan de Fuca area where highly magnetized flows are likely to produce large anomalies. In areas like $18^{\circ} \mathrm{S}$ EPR with much smaller magnetization contrasts, paleointensity techniques provide more accurate values of the main geomagnetic field and therefore have greater potential for dating. 


\section{Acknowledgements}

We thank Peter Selkin, Andrei Kosterov and Jeff Gee for their detailed and helpful reviews and comments. We thank Milene Cormier, Michael Perfit, John Chadwick, H. Paul Johnson and John Sinton for discussions, help and providing us with samples. Research supported by The US National Science Foundation and a LamontDoherty Post-doctoral Fellowship (J.C.). LDEO contribution 6113.[R V]

\section{References}

[1] D.M. Christie, Dating the young ocean floor, Nature 367 (1994) 114-115.

[2] K.H. Rubin, J.D. Macdougall, M.R. Perfit, ${ }^{210} \mathrm{Po}-{ }^{210} \mathrm{~Pb}$ dating of recent volcanic eruptions on the sea floor, Nature 368 (1994) 841-844.

[3] K.H. Rubin, J.D. Macdougall, Dating of neovolcanic MORB using $\left({ }^{226} \mathrm{Ra} /{ }^{230} \mathrm{Th}\right)$ disequilibrium, Earth Planet. Sci. Lett. 101 (1990) 313-322.

[4] S.J. Goldstein, M.T. Murrell, D.J. Janecky, J.R. Delaney, D.A. Clague, Geochronology and petrogenesis of MORB from the Juan de Fuca and Gorda ridges by 238U-230Th disequilibrium, Earth Planet. Sci. Lett. 107 (1991) 25-41.

[5] J. Bloxham, A. Jackson, Time-dependant mapping of the geomagnetic field at the core-mantle boundary, J. Geophys. Res. 97 (1992) 19537-19563.

[6] R. Merrill, M. McElhinny, P. McFadden, The Magnetic Field of the Earth, Academic Press, London, 1996.

[7] T. Pick, L. Tauxe, Geomagnetic palaeointensities during the Cretaceous normal superchron measured using submarine basaltic glass, Nature 366 (1993) 238-242.

[8] W. Zhou, R. Van der Voo, D.R. Peacor, Single-domain and superparamagnetic titanomagnetite in young oceanfloor basalts with variable Ti-content, Earth Planet. Sci. Lett. 150 (1997) 353-362.

[9] W. Zhou, R. Van der Voo, D.R. Peacor, Preservation of pristine titanomagnetite in older ocean-floor basalts and its significance for paleointensity studies, Geology 27 (1999) 1043-1046.

[10] W. Zhou, R. Van der Voo, D. Peacor, Y. Zhang, Variable Ti-content and grain size of titanomagnetite as a function of cooling rate in very young MORB, Earth Planet. Sci. Lett. 179 (2000) 9-20.

[11] P.K.K. Smith, The identification of single-domain titanomagnetite particles by means of transmission electron microscopy, Can. J. Earth Sci. 16 (1979) 375-381.

[12] T. Pick, L. Tauxe, Characteristics of magnetite in submarine basaltic glass, Geophys. J. Int. 119 (1994) 116-128.

[13] D. Dunlop, O. Ozdemir, Rock Magnetism, Cambridge University Press, Cambridge, 1997, 573 pp.
[14] T. Pick, L. Tauxe, Holocene paleointensities: Thellier experiments on submarine basaltic glass from the East Pacific Rise, J. Geophys. Res. 98 (1993) 17949-17964.

[15] V. Mejia, N.D. Opdyke, M.R. Perfit, Paleomagnetic field intensity recorded in submarine basaltic glass from the East Pacific Rise, the last 69 KA, Geophys. Res. Lett. 23 (1996) 475-478.

[16] D.V. Kent, J. Gee, Magnetic alteration of zero-age oceanic basalt, Geology 24 (1996) 703-706.

[17] C.G. Fox, W.E. Radford, R.P. Dziak, T.-K. Lau, Acoustic detection of a seafloor spreading episode on Juan de Fuca ridge using miliary hydrophone arrays, Geophys. Res. Lett. 22 (1995) 131-134.

[18] R.W. Embley, W.W. Chadwick, D. Clague, D. Stakes, 1998 Eruption of axial volcano: Multibeam anomalies and seafloor observations, Geophys. Res. Lett. 26 (23) (1999) 3425-3428.

[19] J.-M. Auzende, J. Sinton and Scientific Party, NAUDUR explorers discover recent volcanic activity along the East Pacific Rise, EOS Trans. Am. Geophys. Union 75 (601) (1994) 604-605.

[20] J.-M. Auzende, V. Ballu, R. Batiza, D. Bideau, J.-L. Charlou, M.-H. Cormier, Y. Fouquet, P. Geistdoerfer, Y. Lagabrielle, J. Sinton, P. Spadea, Recent tectonic, magmatic, and hydrothermal activity on the East Pacific Rise between $17^{\circ} \mathrm{S}$ and $19^{\circ} \mathrm{S}$ : Submersible observations, J. Geophys. Res. 101 (1996) 17995-18010.

[21] J. Sinton, E. Bergamis, R. Batiza, K. Rubin, T. Gregg, K. Gronvold, S. White, K. MacDonald, C. Van Dover, M.H. Cormier, W. Ryan, M. Aigner-Torres, L. Popels, E. Boyle, A. Shah, W. Jin, Volcanological investigations at superfast spreading: Results from R/V Atlantis Cruise 3-31, Ridge Events 10 (1999) 17-23.

[22] M.T. Juarez, L. Tauxe, The intensity of the time-averaged geomagnetic filed: the last $5 \mathrm{Myr}$, Earth Planet. Sci. Lett. 175 (2000) 169-180.

[23] R.S. Coe, Paleointensities of the Earth's magnetic field determined from Tertiary and Quaternary rocks, J. Geophys. Res. 72 (1967) 3247-3262.

[24] T. Nagata, Y. Arai, K. Momose, Secular variation of the geomagnetic total force during the last 5000 years, J. Geophys. Res. 68 (1963) 5277-5281.

[25] J. Gee, D.V. Kent, Magnetization of axial lavas from the southern East Pacific Rise $\left(14^{\circ}-23^{\circ} \mathrm{S}\right)$ : Geochemical controls on magnetic properties, J. Geophys. Res. 102 (1997) 24873-24886.

[26] H.S. Carslaw, J.C. Jaeger, Conduction of Heat in Solids, Clarendon Press, Oxford, 1959, 509 pp.

[27] R. Coe, C. Grommé, E. Mankinen, Geomagnetic paleointensities from radiocarbon dated lava flows on Hawaii and the question of the Pacific non-dipole low, J. Geophys. Res. 83 (1978) 1740-1756.

[28] M. Prevot, E.A. Mankinen, R.S. Coe, C.S. Gromme, The Steen Mountain (Oregon) geomagnetic polarity transition, 2. Field intensity variations and discussion of reversal models, J. Geophys. Res. 90 (1985) 10417-10448.

[29] W.W. Chadwick, R.W. Embley, C.G. Fox, SeaBeam 
depth changes associated with recent lava flows, CoAxial segment, Juna de fuca Ridge: Evidence for multiple eruptions between 1981-1993, Geophys. Res. Lett. 22 (1995) $167-170$.

[30] A. Shah, M.-H. Cormier, W. Ryan, W. Jin, A. Bradley, D. Yoerger, High Resolution 3-D map of the Earth's magnetic field at the EPR reveals shallow dike outcrops and large-scale void space in extrusive layers, EOS Trans AGU, San Francisco, 1999, p. F1074.

[31] L.J. Perram, K.C. Macdonald, S.P. Miller, Deep-tow magnetics near $20^{\circ} \mathrm{S}$ on the East Pacific Rise: A study of short wavelength anomalies at a very fast spreading center, Mar. Geophys. Res. 12 (1990) 235-245.

[32] H.P. Johnson, M.A. Tivey, Magnetic properties of zeroage oceanic crust; a new submarine lava flow on the Juan de Fuca ridge, Geophys. Res. Lett. 22 (1995) 175-178.

[33] M.A. Tivey, H.P. Johnson, A. Bradley, D. Yoerger, Thickness of a submarine lava flow determined from near-bottom magnetic field mapping by autonomous underwater vehicle, Geophys. Res. Lett. 25 (1998) 805808.

[34] J.M. Sinton, S.M. Smaglik, J.J. Mahoney, K.C. Macdonald, Magmatic processes at superfast spreading mid-ocean ridges: Glass compositional variations along the East Pacific Rise $13-23^{\circ} \mathrm{S}$, J. Geophys. Res. 96 (1991) 6133-6155.

[35] A. Jackson, A. Jonkers, M. Walker, Four centuries of geomagnetic secular variation from historical records, Philos. Trans. R. Soc. Lond. 358 (2000) 957-990.

[36] W.H.F. Smith, D.T. Sandwell, Global sea floor topography from satellite altimetry and ship depth soundings, Science 277 (1997) 1956-1962.

[37] J.D.A. Zijderveld, A.C demagnetization of rocks: Analysis of results, in: D.W. Collinson, K.M. Creer, S.K. Runcorn (Eds.), Methods in Palaeomagnetism, Elsevier, Amsterdam, 1967, pp. 254-286. 